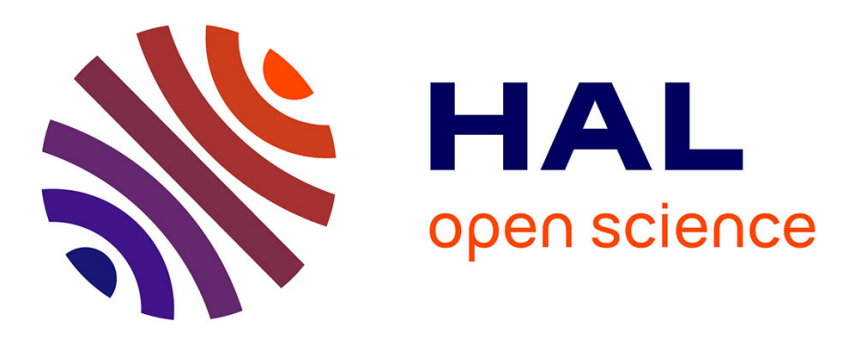

\title{
Improper colouring of weighted grid and hexagonal graphs
}

Jean-Claude Bermond, Frédéric Havet, Florian Huc, Claudia Linhares Sales

\section{To cite this version:}

Jean-Claude Bermond, Frédéric Havet, Florian Huc, Claudia Linhares Sales. Improper colouring of weighted grid and hexagonal graphs. Discrete Mathematics, Algorithms and Applications, 2010, 2 (3), pp.395-411. 10.1142/S1793830910000747 . inria-00526530

\section{HAL Id: inria-00526530 \\ https://hal.inria.fr/inria-00526530}

Submitted on 14 Oct 2010

HAL is a multi-disciplinary open access archive for the deposit and dissemination of scientific research documents, whether they are published or not. The documents may come from teaching and research institutions in France or abroad, or from public or private research centers.
L'archive ouverte pluridisciplinaire HAL, est destinée au dépôt et à la diffusion de documents scientifiques de niveau recherche, publiés ou non, émanant des établissements d'enseignement et de recherche français ou étrangers, des laboratoires publics ou privés. 


\title{
Improper colouring of weighted grid and hexagonal graphs*
}

\author{
Jean-Claude Bermond ${ }^{\dagger} \quad$ Frédéric Havet ${ }^{\dagger} \quad$ Florian Huc $^{\ddagger}$ \\ and Claudia Linhares Sales $\S$
}

August 16, 2010

\begin{abstract}
We study a weighted improper colouring problem motivated by a frequency allocation problem. It consists of associating to each vertex a set of $p(v)$ (weight) distinct colours (frequencies), such that the set of vertices having a given colour induces a graph of degree at most $k$ (the case $k=0$ corresponds to a proper coloring). The objective is to minimize the number of colors. We propose approximation algorithms to compute such colouring for general graphs. We apply these to obtain good approximation ratio for grid and hexagonal graphs. Furthermore we give exact results for the 2-dimensional grid and the triangular lattice when the weights are all the same.
\end{abstract}

\section{Introduction}

This paper is motivated by a problem posed by Alcatel Space Technologies (see [1]). A satellite sends informations to receivers on earth, each of which is listening several frequencies, one for each signal it needs to receive. Technically it is impossible to focus a signal sent by the satellite exactly on the destination receiver. So part of the signal is spread in an area around it, creating noise for the other receivers displayed in this area and listening the same frequency. Each receiver is able to distinguish the signal directed to it from the extraneous noises it picks up if the sum of the noises does not become too large, i.e. does not exceed a certain threshold $T$. The problem is to assign frequencies to the signals in such a way that each receiver gets its dedicated signals properly, while minimizing the total number of frequencies used.

Generally the "noise relation" is symmetric, that is if a receiver $u$ is in the noise area of a receiver $v$ then $v$ is in the noise area of $u$. Hence, interferences may be modelled by a noise graph $G=(V(G), E(G))$ whose vertices are the receivers and where two vertices are joined by an edge if and only if they interfer. Moreover, to the graph is attached a weight function $p: V(G) \rightarrow \mathbb{N}$, where the weight $p(v)$ of the vertex $v$ is equal to the number of signals it has to receive. Hence we have a weighted graph, that is a pair $(G, p)$, where $G$ is a graph and $p$ a weight function on the vertex set of $G$. We can model the frequencies by colours. Therefore, to each vertex, we associate a set $C(v)$ of $p(v)$ distinct colours. If in total $l$ colours are used, the mapping $C$ is called an $l$-colouring of $(G, p)$.

\footnotetext{
${ }^{*}$ This work was partially supported by the INRIA Equipe Associée EWIN

${ }^{\dagger}$ Projet Mascotte I3S (CNRS \& UNS) and INRIA Sophia-Antipolis, 2004 route des Lucioles, BP 93, 06902 SophiaAntipolis Cedex France. E-mail: (bermond|fhavet) @sophia.inria.fr. Partially supported by the ANR Blanc International Taiwan GRATEL.

${ }^{\ddagger}$ Centre Universitaire dInformatique, Battelle bâtiment A, route de Drize 7, 1227 Carouge, Geneva, Switzerland. E-mail: florian.huc@unige.ch. Partially supported by the EU FET project Fronts 215270.

$\S$ Universidade Federal do Ceará, Departamento de Computação, Bloco 910, Campus do Pici, Fortaleza, Ceará, CEP 60455-760, Brasil. linhares@lia.ufc.br
} 
In a simplified version, the intensity $I$ of the noise created by a signal is independent of the frequency and the receiver. Hence to distinguish its signal from noises, a receiver must be in the noise area of at most $k=\left\lfloor\frac{T}{I}\right\rfloor$ receivers listening signals on the same frequency. In terms of colouring this property is equivalent to say that for any colour $c$, the set of vertices having one colour $c$ induces a graph of degree at most $k$.Such a colouring is called $k$-improper. The $k$-improper chromatic number of $(G, p)$, denoted $\chi_{k}(G, p)$, is the smallest $l$ such that $(G, p)$ admits a $k$-improper $l$-colouring. Note that a 0 -improper colouring corresponds to a proper colouring.

In [1] this problem is studied via linear programming. The objective of this paper is to build algorithms giving $k$-improper colourings of weighted graphs of a certain class with as few colours as possible. An algorithm that gives a $k$-improper colouring of each weighted graph $(G, p)$ in this class with at most $c_{1} \times$ $\chi_{k}(G, p)+c_{2}$ colours for some constants $c_{1}$ and $c_{2}$, is said to be $c_{1}$-approximate or to have approximation ratio $c_{1}$.

For any integer $q$ we denote by $\mathbf{q}$ the constant weight function $\mathbf{q}(v)=q$ for all $v$. For any weight function $p$, we set $p_{\max }=\max _{v}(p(v))$.

Proposition 1 If there exists a k-improper colouring of $(G, \mathbf{q})$ with $r$ colours, then there exists a $k$ improper colouring of $(G, p)$ with $r\left\lceil\frac{p_{\max }}{q}\right\rceil$ colours.

In particular, if $\chi_{k}(G, \mathbf{q}) \leq r$, then $\chi_{k}(G, p) \leq r\left\lceil\frac{p_{\max }}{q}\right\rceil$.

Proof. Observe that if we have a $k$-improper $r_{1}$-colouring of $\left(G, p_{1}\right)$ and a $k$-improper $r_{2}$-colouring of $\left(G, p_{2}\right)$, one can easily derive a $k$-improper $\left(r_{1}+r_{2}\right)$-colouring of $\left(G, p_{1}+p_{2}\right)$ by using the union of these colourings on two disjoint sets of colours. Doing this repeatedly $\lambda$ times with $p=\mathbf{q}$, we obtain a $\lambda r$ colouring of $(G, \lambda \mathbf{q})$. For $\lambda=\left\lceil\frac{p_{\max }}{q}\right\rceil$, we obtain a $k$-improper colouring of $(G, p)$ since $p(v) \leq p_{\max } \leq \lambda q$ for every vertex $v \in V(G)$.

If we have a $k$-improper $r$-colouring $(G, \mathbf{q})$ then the above proposition yields an immediate $(r / q)$ approximate algorithm for $k$-improper $(G, p)$-colouring because $\chi_{k}(G, p) \geq p_{\max }$. In this paper, we present improvements of Proposition 1. For any graph $G$, we denote $\rho_{k}(H, G, p)$, the maximum of $\chi_{k}\left(H^{\prime}, p\right)$ over the subgraphs $H^{\prime}$ of $G$ isomorphic to $H$. For example, $\rho_{k}\left(K_{1}, G, p\right)=p_{\max }$ and $\rho_{0}\left(K_{2}, G, p\right)=$ $\max \{p(u)+p(v) \mid u v \in E(G)\}$ so $\rho_{0}\left(K_{2}, G, p\right) \leq 2 p_{\max }$. If $k \geq 1$, then $\rho_{k}\left(K_{2}, G, p\right)=p_{\max }$. By extension, if $\mathcal{H}$ is a family of graphs (finite or not), $\rho_{k}(\mathcal{H}, G, p)$ is the maximum of $\rho_{k}(H, G, p)$ over all graphs $H \in \mathcal{H}$. Obviously, for any family $\mathcal{H}, \rho_{k}(\mathcal{H}, G, p) \leq \chi_{k}(G, p)$. The idea to design approximate algorithms for $k$-improper colouring graphs of some given class consists of finding a finite family of graphs $\mathcal{H}_{k}$ such that any weighted graph $(G, p)$ in the class, satisfies $\chi_{k}(G, p) \leq c_{1} . \rho_{k}\left(\mathcal{H}_{k}, G, p\right)+c_{2}$ with $c_{1}$ a small constant (ideally 1$)$ and $c_{2}$ another constant. Hence, by computing $\chi_{k}\left(H^{\prime}, p\right)$ for all the subgraphs $H^{\prime}$ isomorphic to a graph in $\mathcal{H}_{k}$, we obtain a $c_{1}$-approximate algorithm for $\chi_{k}(G, p)$. Moreover, we also exhibit algorithms that produce the corresponding $c_{1}$-approximate $k$-improper colouring.

In sections 2 and 3 we recall the results known for grid graphs and hexagonal graphs and determine the exact values of $\chi_{k}(G, \mathbf{q}), \mathrm{G}$ being the two-dimensionnal grid or the triangular lattice. Then in Section 4 we give approximate algorithms for general graphs. Finally, in Section 5, we show how to apply these algorithms to get approximations for grid and hexagonal graphs. In the case of grid graphs we improve the approximation ratio for $k=1$. 


\section{Grid graphs}

The (two-dimensionnal) grid is the graph $G L$ defined as follows: the vertices are all integer linear combinations $a \mathbf{f}_{\mathbf{1}}+b \mathbf{f}_{\mathbf{2}}$ of the two vectors $\mathbf{f}_{\mathbf{1}}=(1,0)$ and $\mathbf{f}_{\mathbf{2}}=(0,1)$. Thus, we may identify the vertices with the ordered pairs $(a, b)$ of integers. Two vertices are adjacent when the Euclidean distance between them is 1 . Hence each vertex $x=(a, b)$ has four neighbours: its left neighbour $(a-1, b)$, its right neighbour $(a+1, b)$, its top neighbour $(a, b+1)$ and its down neighbour $(a, b-1)$. A grid graph is an induced subgraph of the two-dimensionnal grid.
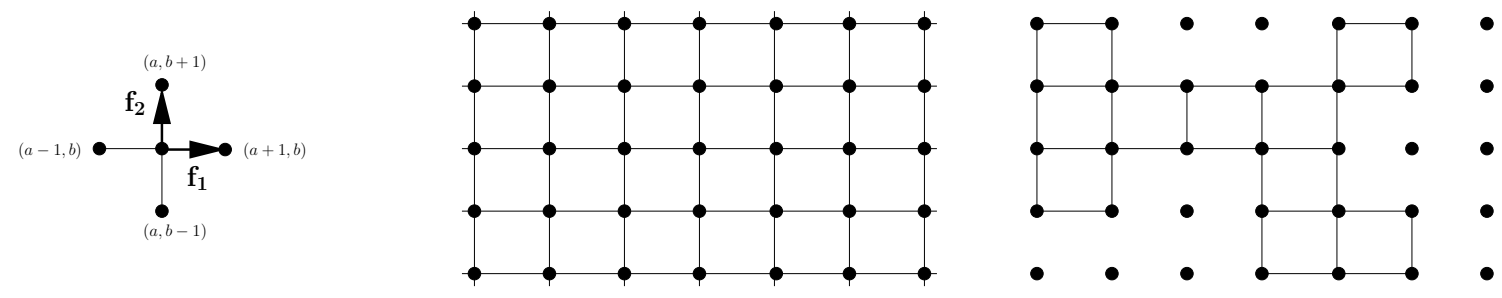

Figure 1: The two-dimensionnal grid and a grid graph.

As a grid graph $G$ has maximum degree $4, k$-improper colourings of $G$ are trivial for $k \geq 4$ : it suffices to give any set of $p(v)$ colours to every vertex $v$ to produce a $k$-improper colouring. Hence, for any $k \geq 4$, its $k$-improper chromatic number equals its maximum weight $\chi_{k}(G, p)=p_{\max }$.

Regarding proper (0-improper) colourings, by Proposition $1, \chi_{0}(G, p) \leq 2 p_{\max }$ as a grid graph is bipartite. This upper bound is tight when there is an edge $u v$ such that $p(u)=p(v)=p_{\max }$. But such an edge may not exist. However, one can find the weighted chromatic number of a grid graph and more generally of any weighted bipartite graph.

Theorem 2 (McDiarmid and Reed [8]) Let $G=((A, B), E)$ be a bipartite graph. Then for any weight $p, \chi_{0}(G, p)=\rho_{0}\left(\left\{K_{1}, K_{2}\right\}, G, p\right)$.

Proof. Let us colour every vertex $a$ of $A$ with $\{1,2, \ldots, p(a)\}$ and every vertex $b$ of $B$ with $\{l, l-$ $1, \ldots, l-p(b)+1\}$ where $l=\rho_{0}\left(\left\{K_{1}, K_{2}\right\}, G, p\right)$.

Now we determine the exact value of $\chi_{k}(G L, \mathbf{q})$ for the two dimensional grid, with constant weight $\mathbf{q}$.

We first prove a preliminary lemma. Let $C$ be a colouring of a weighted graph $(G, p)$. We denote by $c_{u, v}$ the number of colours assigned to both $u$ and $v$, that is $c_{u, v}=|C(u) \cap C(v)|$. We use the standard notation $N(u)$ for the neighbourhood of $u$.

Lemma 3 Let $C$ be a k-improper colouring of a weighted graph $(G, p)$.

(i) $\sum_{v \in N(u)} c_{u, v} \leq k p(u)$;

(ii) $\forall u, v, C$ uses at least $p(u)+p(v)-c_{u, v}$ colours;

(iii) $\forall u, v, w, C$ uses at least $p(u)+p(v)+p(w)-c_{u, v}-c_{u, w}-c_{v, w}$.

Proof. (i) A colour assigned to $u$ is assigned to at most $k$ neighbours of $u$ because $C$ is $k$-improper.

(ii) and (iii) follow from the Inclusion-Exclusion Formula:

$$
|C(u) \cup C(v)|=|C(u)|+|C(v)|-|C(u) \cap C(v)|=p(u)+p(v)-c_{u, v} ;
$$




$$
\begin{aligned}
|C(u) \cup C(v) \cup C(w)|= & |C(u)|+|C(v)|+|C(w)|-|C(u) \cap C(v)|-|C(u) \cap C(w)|-|C(v) \cap C(w)| \\
& +|C(u) \cap C(v) \cap C(w)| \\
\geq & p(u)+p(v)+p(w)-c_{u, v}-c_{u, w}-c_{v, w} .
\end{aligned}
$$

Theorem 4 For the grid GL, we have:

(i) $\chi_{1}(G L, \mathbf{q})=2 q$,

(ii) $\chi_{2}(G L, \mathbf{q})=\left\lceil\frac{3 q}{2}\right\rceil$, and

(iii) $\chi_{3}(G L, \mathbf{q})=\left\lceil\frac{5 q}{4}\right\rceil$.

Proof. Let us first show the $k$-improper colourings of $(G L, \mathbf{q})$ with the required number of colours.

(i) The grid is bipartite so $(G L, \mathbf{q})$ has a 0 -improper (and thus also 1-improper) colouring with $2 q$ colours.

(ii) For $1 \leq j \leq 3$, let $U_{j}=\{(a, b) \mid a+b=j \bmod 3\}$. Assign the colours $\left\lceil\frac{(j-1) q}{2}\right\rceil+1, \ldots,\left\lceil\frac{j q}{2}\right\rceil$ to vertices which are not in $U_{j}$. See Figure 2 for $q=2$.

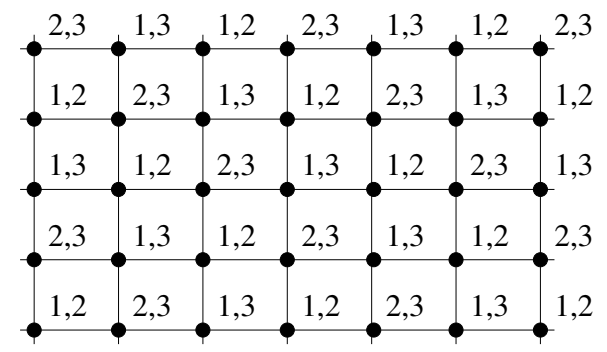

Figure 2: A 2-improper colouring of the square grid.

(iii) For $1 \leq j \leq 5$, let $T_{j}$ be the set of vertices obtained from the vertex $(j, 0)$ by adding the linear combinations of the vectors $2 \mathbf{f}_{\mathbf{1}}+\mathbf{f}_{\mathbf{2}}$ and $5 \mathbf{f}_{\mathbf{1}}$. For $1 \leq j \leq 5$, assign the colours $\left\lceil\frac{(j-1) q}{4}\right\rceil+1, \ldots,\left\lceil\frac{j q}{4}\right\rceil$ to vertices not in $T_{j}$. See Figure 3 for $q=4$.

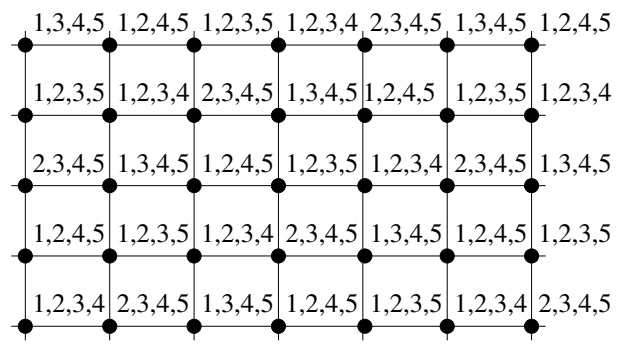

Figure 3: A 3-improper colouring of the square grid. 
Let us now show that these colourings are optimal. To do this, let $C$ be a $k$-improper colouring of $(G L, \mathbf{q})$ with $\chi_{k}(G L, \mathbf{q})$ colours. If $k=1$, consider a 4-cycle in $G L$. A colour may be used on at most two of these vertices. Hence $2 q$ colours are needed. Now, suppose that $2 \leq k \leq 3$ and let $u$ be a vertex of $G L$. Applying Lemma 3 (ii) to the four neighbours of $u$, we obtain: $4 \chi_{k}(G L, \mathbf{q}) \geq 8 q-\sum_{v \in N(u)} c_{u, v}$. Now by Lemma $3(\mathrm{i}), 4 \chi_{k}(G L, \mathbf{q}) \geq(8-k) q$. Therefore, we have respectively $\chi_{2}(G L, \mathbf{q}) \geq \frac{3 q}{2}$ and $\chi_{3}(G L, \mathbf{q}) \geq \frac{5 q}{4}$.

We will use the values given by Theorem 4 to provide in Section 5 , for $1 \leq k \leq 3, \alpha_{k}$-approximate polynomial algorithms that compute a $k$-improper colouring of a weighted grid graph with $\alpha_{1}=13 / 9$, $\alpha_{2}=27 / 20$ and $\alpha_{3}=19 / 16$.

It would be nice to solve the following problem:

Problem 5 For any fixed $1 \leq k \leq 3$, is it $\mathcal{N} \mathcal{P}$-complete to find the $k$-improper chromatic number of a weighted grid graph?

\section{$3 \quad$ Hexagonal graphs}

The triangular lattice graph $T L$ may be described as follows. The vertices are all integer linear combinations $a \mathbf{e}_{\mathbf{1}}+b \mathbf{e}_{\mathbf{2}}$ of the two vectors $\mathbf{e}_{\mathbf{1}}=(1,0)$ and $\mathbf{e}_{\mathbf{2}}=\left(\frac{1}{2}, \frac{\sqrt{3}}{2}\right)$. Thus we may identify the vertices with the ordered pairs $(a, b)$ of integers. Two vertices are adjacent when the Euclidean distance between them is 1 . Therefore, each vertex $x=(a, b)$ has six neighbours: its left neighbour $(a-1, b)$, its right neighbour $(a+1, b)$, its leftup neighbour $(a-1, b+1)$, its rightup neighbour $(a, b+1)$, its leftdown neighbour $(a, b-1)$ and its rightdown neighbour $(a+1, b-1)$. A hexagonal graph is an induced subgraph of the triangular lattice.
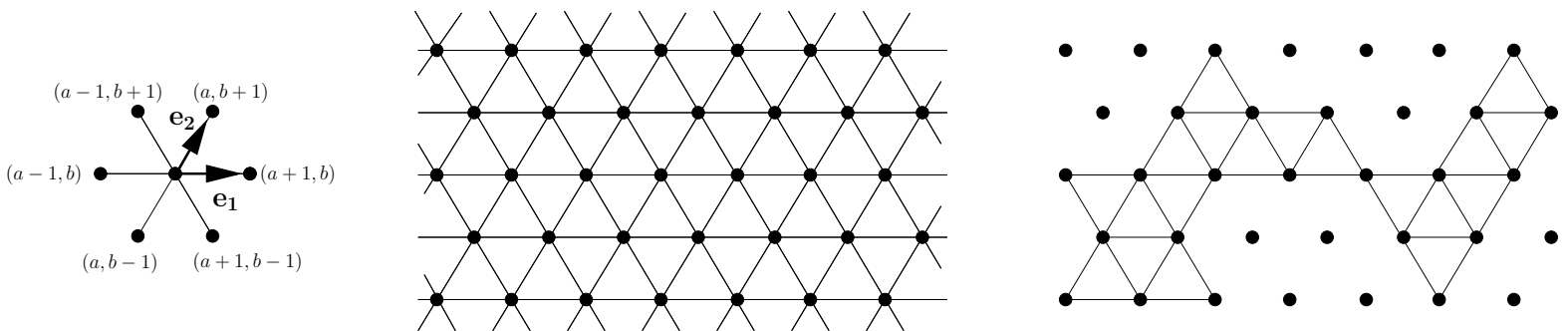

Figure 4: The triangular lattice and a hexagonal graph.

For any $k \geq 6$, the $k$-improper chromatic number of a hexagonal graph is its maximum weight because it has maximum degree 6.

McDiarmid and Reed [8] showed that it is $\mathcal{N} \mathcal{P}$-complete to decide whether the chromatic number of a weighted hexagonal graph is 3 or 4 . Hence, there is no polynomial time algorithm for finding the weighted chromatic number of hexagonal graphs (unless $\mathcal{P}=\mathcal{N} \mathcal{P}$ ). Therefore, one has to find approximate algorithms. The better known so far has approximation ratio $4 / 3$ and is based on the following result:

Theorem 6 (McDiarmid and Reed [8]) For any weigthed hexagonal graph $(G, p)$,

$$
\chi_{0}(G, p) \leq \frac{4}{3} \rho_{0}\left(\left\{K_{1}, K_{2}, K_{3}\right\}, G, p\right) .
$$


A distributed algorithm which guarantees the $\frac{4}{3} \rho_{0}\left(\left\{K_{1}, K_{2}, K_{3}\right\}, G, p\right)$ bound is reported by Narayanan and Schende [7]. However, one expects to have approximate algorithms with ratios better than $4 / 3$. In particular, Reed and McDiarmid conjecture that, for big weights, the ratio may be decreased to almost $9 / 8$.

Conjecture 7 (McDiarmid and Reed [8]) There exists a constant $c$ such that for any weigthed hexagonal graph $(G, p)$,

$$
\chi_{0}(G, p) \leq \frac{9}{8} \rho_{0}\left(\left\{K_{1}, K_{2}, K_{3}\right\}, G, p\right)+c .
$$

Note that the ratio $9 / 8$ in the above conjecture is the best possible. Indeed, consider a 9-cycle $C_{9}$ with constant weight $q$. A colour can be assigned to at most 4 vertices, so $\chi_{0}\left(C_{9}, q\right) \geq \frac{9 q}{4}$. Clearly, $\rho_{0}\left(\left\{K_{1}, K_{2}, K_{3}\right\}, C_{9}, q\right)=2 q$. So $\chi_{0}\left(C_{9}, q\right) \geq \frac{9}{8} \rho_{0}\left(\left\{K_{1}, K_{2}, K_{3}\right\}, C_{9}, q\right)$. An evidence for this conjecture has been given by Havet [2], who proved that if a hexagonal graph $G$ is triangle-free (i.e. has no $K_{3}$ ) then $\chi_{0}(G, p) \leq \frac{7}{6} \rho_{0}\left(\left\{K_{1}, K_{2}\right\}, G, p\right)+5$. See also [10] for an alternative proof and [5] for a distributed algorithm for colouring triangle-free hexagonal graphs with $\frac{5}{4} \rho_{0}\left(\left\{K_{1}, K_{2}\right\}, G, p\right)+3$ colours.

Regarding improper colouring, Havet, Kang and Sereni $[3,4,9]$ generalized the above mentionned $\mathcal{N} \mathcal{P}$-completeness result of McDiarmid and Reed:

Theorem 8 (Havet, Kang and Sereni $[\mathbf{3}, \mathbf{4}, \mathbf{9}]$ ) For $0 \leq k \leq 5$, the following problem is $\mathcal{N} \mathcal{P}$ complete:

Instance: a weighted hexagonal graph $(G, p)$.

Question: is $(G, p)$ k-improper 3-colourable?

Hence one cannot expect to have polynomial algorithm to find the $k$-improper chromatic number of weighted hexagonal graphs.

However, in the particular case of the triangular lattice, we are able to determine the exact values of $\chi_{k}(T L, \mathbf{q})$

Theorem 9 For the triangular lattice TL, we have:

(i) $\chi_{1}(T L, \mathbf{q})=\left\lceil\frac{5 q}{2}\right\rceil$,

(ii) $\chi_{2}(T L, \mathbf{q})=2 q$,

(iii) $\chi_{3}(T L, \mathbf{q})=\left\lceil\frac{3 q}{2}\right\rceil$,

(iv) $\chi_{4}(T L, \mathbf{q})=\left\lceil\frac{4 q}{3}\right\rceil$ and

(v) $\chi_{5}(T L, \mathbf{q})=\left\lceil\frac{7 q}{6}\right\rceil$.

Proof. Let us first show the $k$-improper colourings of $(T L, \mathbf{q})$ with the required number of colours.

(i) For $1 \leq j \leq 5$, let $A_{j}$ be the set of vertices obtained from the vertex $(j, 0)$ by adding the linear combinations of the vectors $2 \mathbf{e}_{1}+\mathbf{e}_{2}$ and $5 \mathbf{e}_{\mathbf{1}}$. For $1 \leq j \leq 5$, assign the colours $\left\lceil\frac{(j-1) q}{2}\right\rceil+1, \ldots,\left\lceil\frac{j q}{2}\right\rceil$ to vertices of $A_{j} \cup A_{j+1}$ (with $A_{6}=A_{1}$ ) (see Figure 3 for $q=2$ ).

(ii) Colour a vertex $(a, b)$ with $1, \ldots, q$ if $a$ is even, and with $q+1, \ldots, 2 q$, otherwise.

(iii) For $1 \leq j \leq 3$, let $S_{j}$ be the set of vertices obtained from the vertex $(j, 0)$, by adding the linear combinations of the vectors $\mathbf{e}_{\mathbf{1}}+\mathbf{e}_{\mathbf{2}}$ and $3 \mathbf{e}_{\mathbf{1}}$. Assign the colours $\left\lceil\frac{(j-1) q}{2}\right\rceil+1, \ldots,\left\lceil\frac{j q}{2}\right\rceil$ to the vertices which are not in $S_{j}$.

(iv) For $0 \leq j_{1} \leq 1$ and $1 \leq j_{2} \leq 2, T_{2 j_{1}+j_{2}}=\left\{(a, b) \mid a \equiv j_{1} \bmod 2\right.$ and $\left.b \equiv j_{2} \bmod 2\right\}$. Assign the colours $\left\lceil\frac{(j-1) q}{3}\right\rceil+1, \ldots,\left\lceil\frac{j q}{3}\right\rceil$ to the vertices which are not in $T_{j}$. 


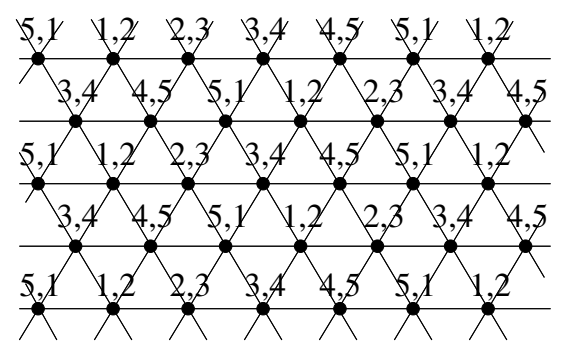

Figure 5: A 1-improper colouring of the triangular lattice.

(v) For $1 \leq j \leq 7$, let $U_{j}$ be the set of vertices obtained from the vertex $(j, 0)$ by adding the linear combinations of the vectors $2 \mathbf{e}_{1}+\mathbf{e}_{2}$ and $7 \mathbf{e}_{\mathbf{1}}$. Assign the colours $\left\lceil\frac{(j-1) q}{6}\right\rceil+1, \ldots,\left\lceil\frac{j q}{6}\right\rceil$ to the vertices which are not in $U_{j}$.

Let us now show that these colourings are optimal. For this, let $C$ be a $k$-improper colouring of $(T L, \mathbf{q})$ with $\chi_{k}(T L, \mathbf{q})$ colours and $u$ be a vertex of $T L$.

Applying Lemma 3 (i) and (ii) to $u$ and its six neighbours, we obtain that $6 \chi_{k}(T L, \mathbf{q}) \geq(12-k) q$. For $k=3,4$ or 5 , we have, respectively, $\chi_{3}(T L, \mathbf{q}) \geq \frac{3 q}{2}, \chi_{4}(T L, \mathbf{q}) \geq \frac{4 q}{3}$ and $\chi_{5}(T L, \mathbf{q}) \geq \frac{7 q}{6}$.

For $1 \leq k \leq 2$, we need an extra argument. Let $u$ and $v$ be two adjacent vertices and $t$ and $w$ their two common neighbours. Set $f(u, v)=c_{u, t}+c_{u, v}+c_{u, w}$.

Consider now the ordered pair $(u, v)$ which minimizes $f(u, v)$, i.e. $f(u, v)=f_{\min }$. Then $f(v, u)=$ $c_{v, t}+c_{u, v}+c_{v, w}$, and therefore, $f(u, v)+f(v, u)=\left(c_{u, t}+c_{u, v}+c_{v, t}\right)+\left(c_{u, w}+c_{u, v}+c_{v, w}\right)$. By Lemma 3 (iii), we have $f(u, v)+f(v, u) \geq 6 q-2 \chi_{k}(T L, \mathbf{q})$.

Let $u^{\prime}$ be the vertex symmetrical to $u$ compared to $v$. By Lemma $3(\mathrm{i}), f(v, u)+f\left(v, u^{\prime}\right) \leq k q$, so $f\left(v, u^{\prime}\right) \leq k q-f(v, u) \leq k q-6 q+2 \chi_{k}(T L, \mathbf{q})+f_{\text {min }}$.

For $k=1$, if $\chi_{k}(T L, \mathbf{q})<\frac{5 q}{2}$, we would have $f\left(v, u^{\prime}\right)<f_{\min }$. Similarly, for $k=2$, if $\chi_{k}(T L, \mathbf{q})<2 q$, we would have $f\left(v, u^{\prime}\right)<f_{\text {min }}$. In both cases, it contradicts the minimality of $f$ in $(u, v)$.

We will use these values to provide in Section 5.2 , for $1 \leq k \leq 5, \alpha_{k}$-approximate polynomial algorithms that compute a $k$-improper colouring of a weighted hexagonal graph with $\alpha_{1}=20 / 11, \alpha_{2}=$ $12 / 7, \alpha_{3}=18 / 13, \alpha_{4}=80 / 63$ and $\alpha_{5}=41 / 36$.

\section{General algorithms}

If $S$ is a set of vertices of $G$, we denote by $G\langle S\rangle$ the subgraph of $G$ induced by the vertices of $S$.

We extend the weight function to sets of vertices and to subgraphs in the following intuitive way: the weight of a set of vertices $X \subset V(G)$ is $p(X)=\sum_{x \in X} p(x)$ while the weight of a subgraph $H \subset G$ is $p(H)=p(V(H))$.

In this section, we improve Proposition 1 . To do so, instead of considering only $p_{\max }$, we consider the number of colours that a vertex and its neighbours may require. As shown by the following proposition, this number may be larger than $p_{\max }$. The graph $K_{1, k+1}$ is the graph with $k+2$ vertices and $k+1$ edges linking one vertex, called the centre to the $k+1$ others, called spikes.

Proposition 10 For every weight function $p, \chi_{k}\left(K_{1, k+1}, p\right) \geq \frac{1}{k+1} \sum_{v \in V\left(K_{1, k+1}\right)} p(v)=\frac{p\left(K_{1, k+1}\right)}{k+1}$. 
Proof. Let $u$ be the centre of $K_{1, k+1}$ and $v_{1}, \ldots, v_{k+1}$ its spikes. Consider a $k$-improper colouring $C$ of $K_{1, k+1}$. For $1 \leq i \leq k+1$, set $q\left(v_{i}\right)=\left|C\left(v_{i}\right) \backslash C(u)\right|$. The colouring $C$ uses at least $M=$ $\max _{1 \leq i \leq k+1}\left\{q\left(v_{i}\right)+p(u)\right\} \geq p(u)+\frac{1}{k+1} \sum_{i=1}^{k+1} q\left(v_{i}\right)$ colours. But a colour in $C(u)$ is assigned to at most $k$ of the spikes because the colouring is $k$-improper. Thus $\sum_{i=1}^{k+1} q\left(v_{i}\right) \geq \sum_{i=1}^{k+1} p\left(v_{i}\right)-k p(u)$. It follows $M \geq \frac{1}{k+1}\left(p(u)+\sum_{i=1}^{k+1} p\left(v_{i}\right)\right)$.

We call $(k+1)$-star, or simply star, a subgraph of $G$ isomorphic to $K_{1, k+1}$.

We set $\theta_{k}(G, p)=\max \{p(H) /(k+1) \mid H$ is a $(k+1)$-star of $G\}$ and $\omega_{k}(G, p)=\max \left\{p_{\max }, \theta_{k}(G, p)\right\}$. According to Proposition 10, $\omega_{k}(G, p) \leq \rho_{k}\left(\left\{K_{1}, K_{1, k+1}\right\}, G, p\right) \leq \chi_{k}(G, p)$.

The idea of our general algorithm is to, step by step, decrease $\omega_{k}(G, p)$ by priorizing the colouring of the vertices with high weights, which directly affect this parameter. Once fixed a $k$-improper colouring $C$ of $(G, \mathbf{q})$ with $r$ colours, at each round of the algorithm, $C$ is repeated several times, spending so, at each round, a multiple of $r$ new colours, in order to assign packets of $q$ colours to these heavy vertices. Also, the colouring of the vertices with small weights is postponed, in such way that, at the last step, the number of colours required to finish the $k$-improper colouring of $G$ is under control. The next theorem gives this general algorithm.

Theorem 11 Let $G$ be a graph with bounded degree $\Delta$ fixed. Let $C$ be a k-improper colouring $C$ of $(G, \mathbf{q})$ with $r$ colours, for some integer $q$. Let $a_{k}(r, q)=(k+1) r-q, \alpha_{k}(r, q)=\frac{a_{k}(r, q) \cdot r+r q}{a_{k}(r, q) \cdot q+r q}=\frac{(k+1) r^{2}}{(k+2) r q-q^{2}}$, $\gamma_{k}(r, q)=\max \left\{(k+2)(r q-1),(k+1) r q+k q^{2}, a_{k}(r, q) q+r q\right\}$ and $\beta_{k}(r, q)=r\left\lceil\frac{\gamma_{k}(r, q)}{q}\right\rceil$.

There is a polynomial algorithm which returns a k-improper colouring of $(G, p)$ with at most $\alpha_{k}(r, q) \times$ $\omega_{k}(G, p)+\beta_{k}(r, q)$ colours.

In particular, if $\chi_{k}(G, \mathbf{q}) \leq r$, then $\chi_{k}(G, p) \leq \alpha_{k}(r, q) \times \omega_{k}(G, p)+\beta_{k}(r, q)$.

Proof. To make the proof more readable, we omit the parameters $k, q$ and $r$ which are fixed. So $a=a_{k}(r, q), \alpha=\alpha_{k}(r, q), \gamma=\gamma_{k}(r, q), \beta=\beta_{k}(r, q)$ and $\omega(G, p)=\omega_{k}(G, p)$. Consider the following algorithm :

Algorithm 1 0. Initialisation: $\left(G^{0}, p^{0}\right):=(G, p), S:=\emptyset$ and $i=0$.

1. Add the vertices of low weight to $S$ that will be treated at the end (Step 3): $S^{i}:=\left\{v \in V(G) \mid p^{i}(v) \leq \gamma\right\}, S:=S \cup S^{i}$ and for all $v \in S^{i}, s(v):=p^{i}(v) . G^{i+1}:=G^{i}-S^{i}$.

2. If $G^{i+1}$ is not empty:

2.1. Give to each vertex $v$ of $G^{i+1}$ a certain number $n^{i}(v)$ of colours among a set of $a r+r q$ colours, in such a way that $\omega\left(G^{i+1}, p^{i}-n^{i}\right) \leq \omega\left(G^{i}, p^{i}\right)-(a q+r q)$.

2.2. Set $p^{i+1}:=p^{i}-n^{i}$ and $i:=i+1$ and go to Step 1 .

3. Colour $(G\langle S\rangle, s)$ with $\beta$ colours. This is possible by Proposition 1 using $\left\lceil\frac{\gamma}{q}\right\rceil$ times the colouring $C$, since $s_{\max } \leq \gamma$.

At each Step 2, ar $+r q$ colours are used and $\omega\left(G^{i}, p^{i}\right)$ decreases by at least $a q+r q$. So after $m=\left\lfloor\frac{\omega(G, p)}{a q+r q}\right\rfloor$ steps, the remaining $\omega\left(G^{m}, p^{m}\right)$ is at most $a q+r q \leq \gamma$ (by the choice of $\gamma$ ). Therefore, Algorithm 1 yields a $k$-improper colouring of $(G, p)$ with at most $\alpha \omega(G, p)+\beta$ colours and the theorem will be proved if we can perform step 2.1 as indicated.

Let us now describe precisely Step 2.1. Set $\omega^{i}=\omega_{k}\left(G^{i}, p^{i}\right)$. We distinguish several kinds of vertices depending on their own weight and the one of their neighbours. A big vertex is a vertex such that 
$p^{i}(v)>\omega^{i}-r q$. A small vertex is a non-big vertex. Moreover, a small vertex is goofy if it is adjacent to a big vertex, and regular, otherwise.

At each step 2.1, we first use $a$ times the colouring $C$ : with $a r$ colours, each vertex receives $a q$ of them. Then we use $r q$ additional colours in the following way: we assign all of them to the big vertices, and we use $q$ times colouring $C$ on the graph induced by the regular vertices. As a result, each big vertex receives $r q$ additional colours and each regular vertex receives $q^{2}$ ones. Hence $n^{i}(v)=a q+r q$ if $v$ is big, $n^{i}(v)=a q+q^{2}$ if $v$ is regular, and $n^{i}(v)=a q$, if $v$ is goofy.

Let us first check that this colouring is $k$-improper.

Suppose by way of contradiction that it is not $k$-improper. The only possibility is that some additional colour has been assigned to a vertex $v$ and $k+1$ of its neighbours since otherwise we were using $C$ which is $k$-improper. Hence $v$ is the centre of a star $H$ whose vertices are all big. Then $p^{i}(H) \geq(k+2)\left(\omega^{i}-r q+1\right)=$ $(k+1) \omega^{i}+\omega^{i}-(k+2)(r q-1)$. But, since $G^{i+1}$ is not empty, $\omega^{i} \geq p_{\max }^{i}>\gamma \geq(k+2)(r q-1)$. So $p^{i}(H)>(k+1) \omega^{i}$ which contradicts the definition of $\omega^{i}$.

Let us now check that $\omega^{i+1} \leq \omega^{i}-(a q+r q)$.

For a vertex $v, p^{i+1}(v) \leq p^{i}(v)-n^{i}(v)$. If $v$ is big $n^{i}(v)=a q+r q$ and, if $v$ is small, $n^{i}(v) \geq a q$ and $p^{i}(v) \leq \omega^{i}-r q$. In both cases, $p^{i+1}(v) \leq \omega^{i}-(a q+r q)$.

So $p_{\max }^{i+1} \leq \omega^{i}-(a q+r q)$.

Consider now a star $H$ of $G^{i+1}$. It is big if $p^{i}(H) \geq(k+1) \omega^{i}-q^{2}$.

Claim 1 A big star $H$ has a vertex which is not goofy.

Proof. By the contrapositive. Let $H$ be a star whose vertices are all goofy. Let $u$ be its centre and $v_{1}, \ldots, v_{k+1}$ its spikes with $p^{i}\left(v_{1}\right) \geq \cdots \geq p^{i}\left(v_{k+1}\right)$.

There exists a big vertex $v_{0}$ adjacent to $u$. The vertex $v_{0}$ is not one of the $v_{i}$, because all of them are small. Let $H^{\prime}$ be the star with centre $u$ and spikes $v_{0}, \ldots, v_{k}$. Set $S=\sum_{j=1}^{k} p^{i}\left(v_{j}\right)$. We have $p^{i}\left(H^{\prime}\right)=p^{i}(u)+S+p^{i}\left(v_{0}\right) \leq(k+1) \omega^{i}$ and $p^{i}\left(v_{0}\right)>\omega^{i}-r q$. Hence $S<k \omega^{i}+r q-p^{i}(u)$. But $p^{i}\left(v_{k+1}\right) \leq \frac{S}{k}$, so $p^{i}\left(v_{k+1}\right)<\omega^{i}+\frac{r q}{k}-\frac{p^{i}(u)}{k}$.

Observe that $p^{i}(H)=p^{i}(u)+S+p^{i}\left(v_{k+1}\right)$ and so, by consequence of the above inequalities, $p^{i}(H)<$ $(k+1) \omega^{i}+r q\left(\frac{k+1}{k}\right)-\frac{p^{i}(u)}{k}$. As $u \in G^{i+1}$, we have $p^{i}(u)>\gamma \geq(k+1) r q+k q^{2}$, thus $p^{i}(H)<(k+1) \omega^{i}-q^{2}$. Hence $H$ is not big.

Each vertex of $H$ receives at least $a q$ colours. Hence if $H$ is not big, $p^{i+1}(H) \leq p^{i}(H)-(k+2) a q \leq$ $(k+1) \omega^{i}-q^{2}-(k+2) a q$. If $H$ is big, at least one vertex of $H$ is not goofy and so receives at least $a q+q^{2}$ colours. Thus in both cases

$$
p^{i+1}(H) \leq(k+1) \omega^{i}-(k+2) a q-q^{2} \leq(k+1)\left(\omega^{i}-(a q+r q)\right),
$$

because $a=(k+1) r-q$.

Thus $\theta_{k}\left(G^{i+1}, p^{i+1}\right) \leq \omega^{i}-(a q+r q)$. Hence, since $p_{\max }^{i+1} \leq \omega^{i}-(a q+r q)$, we get $\omega^{i+1} \leq \omega^{i}-(a q+r q)$.

Algorithm 1 requires to compute the value of $\omega_{k}(G, p)$. Since there are at most $n\left(\begin{array}{c}\Delta \\ k+1\end{array}\right) k+1$-stars in a graph on $n$ vertices with maximum degree $\Delta$, it can be done in $O\left(n\left(\begin{array}{c}\Delta \\ k+1\end{array}\right)\right)$ operations.

For 1-improper colouring, changing slightly the parameters of Algorithm 1 yields a better approximation ratio. Replacing $\beta_{1}(r, q)$ by a slightly higher value $\beta_{1}^{\prime}(r, q)$ allows us to replace $a_{1}(r, q)$ by a smaller value $a_{1}^{\prime}(r, q)$ which yields a better approximation ratio $\alpha_{1}^{\prime}(r, q)=\frac{a_{1}^{\prime}(r, q) \cdot r+r q}{a_{1}^{\prime}(r, q) \cdot q+r q}$. 
Theorem 12 Let $r$ and $q$ be two integers. Set $a_{1}^{\prime}(r, q)=2 r-2 q$ if $r \geq 2 q, a_{1}^{\prime}(r, q)=r$ if $r \leq 2 q$, $\alpha_{1}^{\prime}(r, q)=\frac{a_{1}^{\prime}(r, q) \cdot r+r q}{a_{1}^{\prime}(r, q) \cdot q+r q}, \gamma_{1}^{\prime}(r, q)=3 r q$ and $\beta_{1}^{\prime}(r, q)=3 r^{2}$. There is a polynomial algorithm that, given a weighted graph $G$ and a 1-improper colouring $C$ of $(G, \mathbf{q})$ with $r$ colours, produces a 1-improper colouring of $(G, p)$ with at most $\alpha_{1}^{\prime}(r, q) \times \omega_{1}(G, p)+\beta_{1}^{\prime}(r, q)$ colours.

In particular, if $\chi_{1}(G, \mathbf{q}) \leq r$, then $\chi_{1}(G, p) \leq \alpha_{1}^{\prime}(r, q) \times \omega_{1}(G, p)+\beta_{1}^{\prime}(r, q)$.

Proof. We use Algorithm 1 with $\beta=\beta_{1}^{\prime}(r, q), a=a_{1}^{\prime}(r, q), \alpha=\alpha_{1}^{\prime}(r, q)$ and $\gamma=\gamma_{1}^{\prime}(r, q)$.

Note that $\gamma \geq 3 r q$ implies $\gamma \geq a q+r q$ as $a q+r q=3 r q-2 q^{2}$ if $r \geq 2 q$ and $a q+r q=2 r q$ if $r \leq 2 q$.

The beginning of the proof is the same as that of Theorem 11. Note that the condition needed for the $k$-improper property is $\gamma \geq 3(r q-1)$ which is satisfied. Like in Theorem 11, we have $p_{\max }^{i+1} \leq \omega^{i}-(a q+r q)$. (recall that $\left.\omega^{i}=\omega_{1}\left(G^{i}, p^{i}\right)\right)$. So in order to show that $\omega^{i+1} \leq \omega^{i}-(a q+r q)$, it remains to show that $\theta_{k}\left(G^{i+1}, p^{i+1}\right) \leq \omega^{i}-(a q+r q)$, that is for any 2 -star $H$ of $G^{i+1}, \rho^{i+1}(H) \leq 2\left(\omega^{i}-(a q+r q)\right)$.

For that let us redefine a big star in $G^{i}$ as a 2 -star such that $p^{i}(H)>2 \omega^{i}-2 r q+a q$.

Claim 2 A big star contains either a big vertex or two regular vertices.

Proof. Suppose $H$ is a 2 -star with centre $u$ and spikes $v_{1}$ and $v_{2}$. Assume, moreover, that two of these vertices are goofy and none is big. W.l.o.g. there is a big vertex $b_{1}$ adjacent to $v_{1}$ and a big vertex $b_{2}$ adjacent to $u$ or $v_{2}$. As $b_{1}, v_{1}$ and $u$ form a 2-star, by the definition of $\omega^{i}, p^{i}\left(b_{1}\right)+p^{i}\left(v_{1}\right)+p^{i}(u) \leq 2 \omega^{i}$. Similarly, $p^{i}\left(b_{2}\right)+p^{i}(u)+p^{i}\left(v_{2}\right) \leq 2 \omega^{i}$. Summing these two inequalities, we obtain $p^{i}\left(v_{1}\right)+p^{i}(u)+p^{i}\left(v_{2}\right) \leq$ $4 \omega^{i}-p^{i}\left(b_{1}\right)-p^{i}\left(b_{2}\right)-p^{i}(u) \leq 2 \omega^{i}-p^{i}(u)+2 r q-2$. Since $u$ is in $G^{i+1}, p^{i}(u) \geq \gamma \geq 4 r q-a q-1$ because $4 r q-a q=2 r q+2 q^{2} \leq 3 r q$ if $r \geq 2 q$, and $4 r q-a q=3 r q$ if $r \leq 2 q$. So $p^{i}(H) \leq 2 \omega^{i}-2 r q+a q$.

Each vertex receives at least $a q$ colours. Hence, if $H$ is not big, then $p^{i+1}(H) \leq p^{i}(H)-3 a q \leq$ $2 \omega^{i}-2 r q-2 a q$

Suppose now that $H$ is big. Then, by Claim 2, $H$ contains a big vertex or two regular ones. Hence $p^{i+1}(H) \leq p^{i}(H)-3 a q-\min \left\{r q, 2 q^{2}\right\}$. By our choice of $a$, we get $p^{i+1}(H) \leq 2\left(\omega^{i}-(a q+r q)\right)$.

When $\Delta(G)=k+1$, one can also get a better approximation ratio. To do so, we need to change the parameters $a_{k}(r, q), \alpha_{k}(r, q)$ and $\beta_{k}(r, q)$ but also to modify slightly Step 2.1 of Algorithm 1. We take advantage of the condition $\Delta(G)=k+1$ to more precisely allow the $r q$ additional colours.

Theorem 13 Let $r$ and $q$ be two integers. Set $a_{k}^{\prime \prime}(r, q)=(k+1) r-2 q$ if $r \geq 2 q$, and $a_{k}^{\prime \prime}(r, q)=k r$ if $r \leq 2 q$, and $\alpha_{k}^{\prime \prime}(r, q)=\frac{a_{k}^{\prime \prime}(r, q) \cdot r+r q}{a_{k}^{\prime \prime}(r, q) \cdot q+r q}, \gamma_{k}^{\prime \prime}(r, q)=\max \left\{(k+2)(r q-1), a_{k}^{\prime \prime}(r, q) q+r q\right\}$ and $\beta_{k}^{\prime \prime}(r, q)=$ $r\left\lceil\frac{\gamma^{\prime \prime}(r, q)}{q}\right\rceil$. There exists a polynomial algorithm that given a graph $G$ with maximum degree $k+1$ and a $k$-improper colouring $C$ of $(G, \mathbf{q})$ with $r$ colours, produces a k-improper colouring of $(G, p)$ with at most $\alpha_{k}^{\prime \prime}(r, q) \times \omega_{k}(G, p)+\beta_{k}^{\prime \prime}(r, q)$ colours.

In particular, if $\chi_{k}(G, \mathbf{q}) \leq r$, then $\chi_{k}(G, p) \leq \alpha_{k}^{\prime \prime}(r, q) \times \omega_{k}(G, p)+\beta_{k}^{\prime \prime}(r, q)$.

Proof. We use an algorithm globally identical to Algorithm 1 with $\beta=\beta_{k}^{\prime \prime}(r, q), a=a_{1}^{\prime \prime}(r, q), \gamma=\gamma_{k}^{\prime \prime}(r, q)$ and $\alpha=\alpha_{1}^{\prime \prime}(r, q)$ in place of $\beta_{k}(r, q), a_{k}(r, q)$ and $\alpha_{k}(r, q)$ respectively.

Only Step 2.1 is slightly modified in the following way.

As before $\omega^{i}=\omega_{k}\left(G^{i}, p^{i}\right)$. Big, small, goofy and regular vertices are defined as in the proof of Theorem 11. A regular vertex is isolated if it is adjacent to no regular vertex.

As in the proof of Theorem 11, at each Step 2.1, we first use a times the colouring $C$ : with ar colours, each vertex receives $a q$ of them. But the $r q$ additional colours are assigned in a slightly different way: we give all of them to the big and isolated regular vertices, and we use $q$ times colouring $C$ on the non-isolated regular vertices, so that each them get $q^{2}$ additional colours. Hence $n^{i}(v)=a q+r q$ if $v$ is big or isolated regular, $n^{i}(v)=a q+q^{2}$ if $v$ is non-isolated regular, and $n^{i}(v)=a q$ if $v$ is goofy. 
One shows that the obtained colouring is $k$-improper in the same way as in the proof of Theorem 11 . We need to check that for each $i, \omega^{i+1} \leq \omega^{i}-(a q+r q)$.

Identically to the proof of Theorem 11 , as $\gamma \geq(k+2)(r q-1)$, one shows that $p_{\max }^{i+1} \leq \omega^{i}-(a q+r q)$.

Claim $3 A$ star $H$ contains a big vertex or an isolated regular vertex $x$ or two non-isolated regular vertices.

Proof. Let $u$ be the centre of $H$ and $v_{1}, \ldots, v_{k+1}$ its spikes. Since $G$ has maximum degree $k+1$ the only neighbours of $u$ are the $v_{i}$ 's. Hence, if no vertex of $H$ is big, then $u$ is regular. Moreover, if $u$ is not isolated, one of the $v_{i}$ 's is also regular.

Consider a star $H$ of $G^{i+1}$. Observe that each vertex of $H$ receives at least $a q$ colours. Moreover, by Claim 3, $H$ contains a vertex that receives at least $a q+r q$ colours or two vertices receiving at least $a q+q^{2}$ colours each. Consequently, $p^{i+1}(H) \leq p^{i}(H)-(k+2) q a-\min \left\{r q, 2 q^{2}\right\}$. By our choice of $a$, we get $p^{i+1}(H) \leq(k+1)\left(\omega^{i}-(a q+r q)\right)$.

Thus $\theta_{k}\left(G^{i+1}, p^{i+1}\right) \leq \omega^{i}-a q-r q$, and so $\omega^{i+1} \leq \omega^{i}-a q-r q$.

\section{Applications to grid graphs and hexagonal graphs}

\subsection{Grid graphs}

We now apply Theorems 11, 12 and 13 to grid graphs, by using the values $\chi_{k}(G L, \mathbf{q})$ determined in Theorem 4.

Theorem 14 For $1 \leq k \leq 3$, there are $\alpha_{k}$-approximate algorithms for finding a $k$-improper colouring of a weighted grid graph, where $\alpha_{1}=\frac{3}{2}, \alpha_{2}=\frac{27}{20}$, and $\alpha_{3}=\frac{19}{16}$.

Proof. Theorems 12 and 4 give the result for $k=1$. Theorems 11 and 4 give the result for $k=2$. Theorems 13 and 4 give the result for $k=3$.

The following theorem improves the last result for 1-improper colouring of weighted grid graphs:

Theorem 15 There is a $\frac{13}{9}$-approximate algorithm for finding a 1-improper colouring of weighted grid graph.

Proof. The general idea of the algorithm is still similar, but this time we take advantage of the position of big vertices and quasi-big vertices (a new type of vertex to be described) to 1-improper colour the grid graph (cf Claim 4).

Algorithm $2 \quad 0$. Initialisation: $\left(G^{0}, p^{0}\right):=(G, p), S:=\emptyset$ and $i=0$.

1. Add the vertices of low weight to $S$ that will be treated at the end (Step 3):

$S^{i}:=\left\{v \in V(G) \mid p^{i}(v)<p_{\text {inf }}\right\}$ with $p_{\text {inf }}=133, S:=S \cup S^{i}$ and for all $v \in S^{i}, s(v):=p^{i}(v)$. $G^{i+1}:=G^{i}-S^{i}$.

2. If $G^{i+1}$ is not empty:

2.1. Give to each vertex $v$ of $G^{i+1}$ a certain number $n^{i}(v)$ of colours among a set of 156 colours, in such a way that $\omega_{k}\left(G^{i+1}, p^{i}-n^{i}\right) \leq \omega_{k}\left(G^{i}, p^{i}\right)-108$.

2.2. Set $p^{i+1}:=p^{i}-n^{i}$ and $i:=i+1$, and go to Step 1 . 
3. Colour $(G\langle S\rangle, s)$ with 264 colours. It is possible as $s_{\max } \leq p_{\text {inf }}-1=132$ and $\chi_{1}(G L, q)=2 q$.

Algorithm 2 yields a 1-improper colouring of $(G, p)$ with at most $\frac{156}{108} \omega_{k}(G, p)+264$ colours.

Here again we set $\omega^{i}=\omega_{1}\left(G^{i}, p^{i}\right)$. Let us now describe precisely how to perform Step 2.1. A big vertex is a vertex such that $p^{i}(v)>\omega^{i}-d_{1}$ with $d_{1}=24$. A small vertex $v$ is a non-big vertex. It is goofy if it is adjacent to a big vertex (we note Goof the set of goofy vertices), it is quasi-big if $p^{i}(v)>\omega^{i}-d_{2}$ with $d_{2}=67$, and it is regular, otherwise. A 2-star is big if its weight is bigger than $2 \omega^{i}-d_{3}$ with $d_{3}=45$.

Claim 4 Let $u$ and $v$ be two vertices which are big or quasi-big in $G^{i}$. In $G^{i+1}$, they are at distance at least 3 or form a connected component.

Proof. Suppose that $u$ and $v$ are at distance at most 2. If they do not form a connected component in $G^{i+1}$, there is a vertex $w \in V\left(G^{i+1}\right)$ such that the subgraph induced by $u, v$ and $w$ is a 2-star. Hence $p^{i}(u)+p^{i}(v)+p^{i}(w) \leq 2 \omega^{i}$. But $p^{i}(u)+p^{i}(v)+p^{i}(w)>2 \omega^{i}-2 d_{2}+1+p_{\text {inf }}=2 \omega^{i}$, a contradiction. Thus $u$ and $v$ form a connected component.

Set $a=48, b=6$ and $c=3$. At each step 2.1, we give all the $2 a+8 b+4 c$ colours to the vertices in the connected components of order 2 .

For the vertices not in such small components, we use three different colourings. The two first are based on the following eight sets of vertices:

- $U_{1}=\left\{(0,0)+2 i \mathbf{f}_{\mathbf{1}}+i^{\prime}\left(\mathbf{f}_{\mathbf{1}}-2 \mathbf{f}_{\mathbf{2}}\right),(0,1)+2 i \mathbf{f}_{\mathbf{1}}+i^{\prime}\left(\mathbf{f}_{\mathbf{1}}-2 \mathbf{f}_{\mathbf{2}}\right)\right\}$,

- $U_{2}=\left\{(1,0)+2 i \mathbf{f}_{\mathbf{1}}+i^{\prime}\left(\mathbf{f}_{\mathbf{1}}-2 \mathbf{f}_{\mathbf{2}}\right),(1,1)+2 i \mathbf{f}_{\mathbf{1}}+i^{\prime}\left(\mathbf{f}_{\mathbf{1}}-2 \mathbf{f}_{\mathbf{2}}\right)\right\}$,

- $U_{3}=\left\{(0,1)+2 i \mathbf{f}_{\mathbf{1}}+i^{\prime}\left(\mathbf{f}_{\mathbf{1}}-2 \mathbf{f}_{\mathbf{2}}\right),(0,2)+2 i \mathbf{f}_{\mathbf{1}}+i^{\prime}\left(\mathbf{f}_{\mathbf{1}}-2 \mathbf{f}_{\mathbf{2}}\right)\right\}$,

- $U_{4}=\left\{(1,1)+2 i \mathbf{f}_{\mathbf{1}}+i^{\prime}\left(\mathbf{f}_{\mathbf{1}}-2 \mathbf{f}_{\mathbf{2}}\right),(1,2)+2 i \mathbf{f}_{\mathbf{1}}+i^{\prime}\left(\mathbf{f}_{\mathbf{1}}-2 \mathbf{f}_{\mathbf{2}}\right)\right\}$,

- $U_{5}=\left\{(0,0)+2 i \mathbf{f}_{\mathbf{2}}+i^{\prime}\left(2 \mathbf{f}_{\mathbf{1}}-\mathbf{f}_{\mathbf{2}}\right),(1,0)+2 i \mathbf{f}_{\mathbf{2}}+i^{\prime}\left(2 \mathbf{f}_{\mathbf{1}}-\mathbf{f}_{\mathbf{2}}\right)\right\}$,

- $U_{6}=\left\{(0,1)+2 i \mathbf{f}_{\mathbf{2}}+i^{\prime}\left(2 \mathbf{f}_{\mathbf{1}}-\mathbf{f}_{\mathbf{2}}\right),(1,1)+2 i \mathbf{f}_{\mathbf{2}}+i^{\prime}\left(2 \mathbf{f}_{\mathbf{1}}-\mathbf{f}_{\mathbf{2}}\right)\right\}$,

- $U_{7}=\left\{(1,0)+2 i \mathbf{f}_{\mathbf{2}}+i^{\prime}\left(2 \mathbf{f}_{\mathbf{1}}-\mathbf{f}_{\mathbf{2}}\right),(2,0)+2 i \mathbf{f}_{\mathbf{2}}+i^{\prime}\left(2 \mathbf{f}_{\mathbf{1}}-\mathbf{f}_{\mathbf{2}}\right)\right\}$,

- $U_{8}=\left\{(1,1)+2 i \mathbf{f}_{\mathbf{2}}+i^{\prime}\left(2 \mathbf{f}_{\mathbf{1}}-\mathbf{f}_{\mathbf{2}}\right),(2,1)+2 i \mathbf{f}_{\mathbf{2}}+i^{\prime}\left(2 \mathbf{f}_{\mathbf{1}}-\mathbf{f}_{\mathbf{2}}\right)\right\}$.

We first assign $2 a$ colours such that each vertex of $U_{1}$ receive $a$ colours and each vertex of $U_{2}$ receives $a$ other colours. Since $U_{1}$ and $U_{2}$ form a partition of $V$, each vertex receives a colours, and since $G\left\langle U_{1}\right\rangle$ and $G\left\langle U_{2}\right\rangle$ have maximum degree 1 , the colouring we obtain is 1 -improper.

Then we use $8 b$ colours denoted by $(i, j), 1 \leq i \leq b, 1 \leq j \leq 8$. We give all these colours to the big vertices. Then, for $1 \leq j \leq 8$, we give to each small vertex of $U_{j} \backslash$ Goof the colours $(i, j), 1 \leq i \leq b$. A vertex appears in four of the $U_{j}$, so each non-goofy vertex receives $4 b$ colours. Finally, by Claim 4 , a goofy vertex $u$ has a unique big neigbour $v$. Thus, there is an integer $j$ such that both $u$ and $v$ are in $U_{j}$. We assign to $u$ the colours $(i, j), 1 \leq i \leq b$. Doing so, each goofy vertex receives $b$ colours.

Finally, we use four sets of $c$ colours $A_{t}, A_{d}, A_{l}$ and $A_{r}$. Each big or quasi-big vertex receives the colours of all these sets and each top (resp. down, left, right) neighbour receives the colours of $A_{t}$ (resp. $A_{d}, A_{l}$ and $\left.A_{r}\right)$. This is 1-improper, by Claim 4 .

Let us now check that $\omega^{i+1} \leq \omega^{i}-108$. Let $v$ be a vertex. If it is big then $n^{i}(v)=a+8 b+4 c$; if it is quasi-big, then $n^{i}(v)=a+4 b+4 c$; if it is goofy, then $n^{i}(v)=a+b+c$; if it is regular, then $n^{i}(v) \geq a+4 b$, and if, in addition, it is adjacent to a quasi-big vertex, then $n^{i}(v) \geq a+4 b+c$. Hence, by our choice of $a, b, c, d_{1}$ and $d_{2}$, we have

$$
p_{\max }^{i+1} \leq \omega^{i}-\min \left\{a+8 b+4 c, d_{1}+a+4 b+4 c, d_{2}+a+b+c, d_{2}+a+4 b\right\} \leq p_{\max }^{i}-108 .
$$




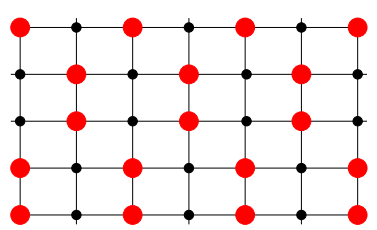

$U_{1}$

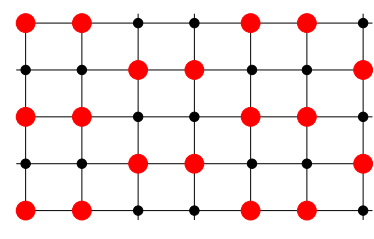

$U_{5}$

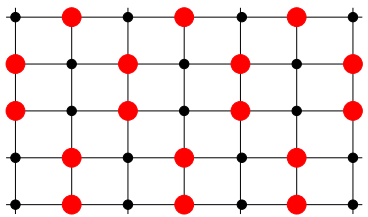

$U_{2}$

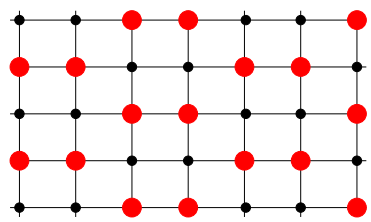

$U_{6}$

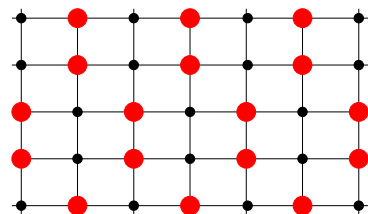

$U_{3}$

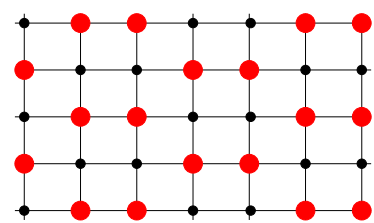

$U_{7}$

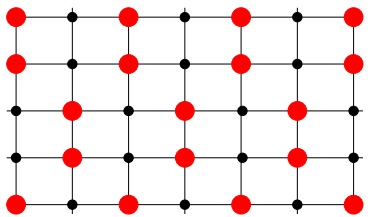

$U_{4}$

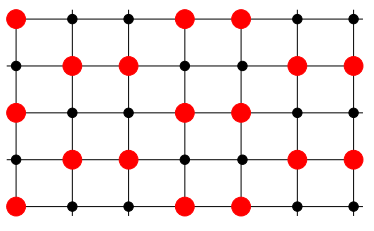

$U_{8}$

Figure 6: The sets $U_{i}, 1 \leq i \leq 8$. In each figure, the bold vertices are those of $U_{i}$ and the bottom left vertex is $(0,0)$.

Claim 5 Let $H$ be a big 2-star in $\left(G^{i+1}, p^{i}\right)$. Then, the following hold:

(i) if the centre of $H$ is goofy, then $H$ has a big vertex;

(ii) if $H$ has two goofy vertices, then the third one is big;

(iii) if $H$ has a goofy vertex, then it has a big or quasi-big vertex.

Proof. Let $u$ be the centre of $H$ and $v_{1}$ and $v_{2}$ its spikes.

(i) Suppose that $u$ is goofy. Let $w$ be its big neighbour. Suppose for a contradiction that $w \notin\left\{v_{1}, v_{2}\right\}$. Considering the two 2 -stars with vertex sets $\left\{w, u, v_{1}\right\}$ and $\left\{w, u, v_{2}\right\}$, we obtain that $p^{i}\left(v_{1}\right)+p^{i}(u) \leq$ $2 \omega^{i}-p^{i}(w) \leq \omega^{i}+d_{1}-1$ and $p^{i}\left(v_{2}\right)+p^{i}(u) \leq 2 \omega^{i}-p^{i}(w) \leq \omega^{i}+d_{1}-1$. Hence $2 \omega^{i}+2 d_{1}-2 \geq$ $p^{i}\left(v_{1}\right)+p^{i}\left(v_{2}\right)+2 p^{i}(u) \geq p^{i}(u)+p^{i}(H) \geq p^{i}(u)+2 \omega^{i}-d_{3}+1$, and so $p^{i}(u) \leq 2 d_{1}+d_{3}-3$. But then $u$ is in $S^{i}$, which is a contradiction.

(ii) Suppose, by way of contradiction, that $H$ has two goofy vertices and no big vertex. By (i), $v_{1}$ and $v_{2}$ are the goofy vertices. Let $w_{1}$ (resp. $w_{2}$ ) be the big neighbour of $v_{1}$ (resp. $v_{2}$ ). (We may have $\left.w_{1}=w_{2}\right)$. As in (i), considering the two 2-stars with vertex sets $\left\{w_{1}, v_{1}, u\right\}$ and $\left\{w_{2}, v_{2}, u\right\}$, we obtain $p^{i}\left(v_{1}\right)+p^{i}(u) \leq \omega^{i}+d_{1}-1$ and $p^{i}\left(v_{2}\right)+p^{i}(u) \leq \omega^{i}+d_{1}-1$. These inequalities yield the contradiction as in (i).

(iii) If $u$ is goofy, then $H$ has a big vertex by (i). Suppose now that one spike of $H$, say $v_{1}$, is goofy. Let $w$ be the big neighbour of $v_{1}$. If $w=u$, we have the result. Assume now that $w \neq u$. Considering the 2-star induced by $\left\{w, v_{1}, u\right\}$, we obtain $p^{i}\left(v_{1}\right)+p^{i}(u) \leq \omega^{i}+d_{1}-1$. Since $H$ is big, $p^{i}\left(v_{1}\right)+p^{i}(u)+p^{i}\left(v_{2}\right) \geq 2 \omega^{i}-d_{3}+1$. So $p^{i}\left(v_{2}\right) \geq \omega^{i}-d_{1}-d_{3}+2$, that is, $v_{2}$ is quasi-big.

Let $H$ be a 2 -star. If it is not big, its weight decreases by at least $3 a+3 b+3 c$. Hence

$$
p^{i+1}(H) \leq 2 \omega^{i}-45-(3 a+3 b+3 c)=2 w^{i}-216
$$

If $H$ is big, then by Claim 5, it has either three non-goofy vertices, in which case its weight decreases by at least $3 a+12 b$, or one big vertex and two goofy vertices, in which case its weight decreases by $3 a+10 b+6 c$, or one big vertex, one regular vertex and one goofy vertex, in which case its weight decreases by $3 a+13 b+5 c$, or one quasi-big vertex, one regular vertex adjacent to this quasi-big vertex 
and one goofy vertex, in which case its weight decreases by $3 a+9 b+6 c$. Hence, by our choice of $a, b, c$, for a big star $H$, we have:

$$
p^{i+1}(H) \leq p^{i}(H)-\min (3 a+12 b, 3 a+9 b+6 c, 3 a+13 b+5 c) \leq p^{i}(H)-216 .
$$

We conclude the proof observing that Inequalities (1), (2) and (3) yield $\omega^{i+1} \leq \omega^{i}-108$.

\subsection{Hexagonal graphs}

We apply Theorems 11, 12 and 13 to hexagonal graphs, with the values $\chi_{k}(T L, \mathbf{q})$ determined in Theorem 9 .

Theorem 16 For $1 \leq k \leq 5$, there are $\alpha_{k}$-approximate algorithms for finding a $k$-improper colouring of a weighted hexagonal graph, where $\alpha_{1}=\frac{20}{11}, \alpha_{2}=\frac{12}{7}, \alpha_{3}=\frac{18}{13}, \alpha_{4}=\frac{80}{63}$, and $\alpha_{5}=\frac{41}{36}$.

Proof. Theorems 12 and 9 give the result for $k=1$. Theorems 11 and 9 give the result for $2 \leq k \leq 4$. Finally, Theorems 13 and 9 give the result for $k=5$.

\section{Conclusion}

We have proposed several approximate algorithms whose approximation ratios are summarized in the Table 1.

\begin{tabular}{|c|c|c|c|c|c|}
\hline & $k=1$ & $k=2$ & $k=3$ & $k=4$ & $k=5$ \\
\hline Grid & $13 / 9$ & $27 / 20$ & $19 / 16$ & 1 & 1 \\
\hline Hexagonal & $20 / 11$ & $12 / 7$ & $18 / 13$ & $80 / 63$ & $41 / 36$ \\
\hline
\end{tabular}

Table 1: Summary of the approximation ratios.

A natural continuation of this work would be to improve the above ratios. It would be very nice to prove the existence of a Polynomial Time Approximation Scheme or some unapproximability results for these problems. In this direction, the first thing to do regarding grid graphs is to answer Problem 5, i.e. to find whether or not optimally improper colouring a weighted grid graph is NP-complete. We strongly believe that it must be NP-complete.

\section{References}

[1] S. Alouf, E. Altman, J. Galtier, J.-F. Lalande, and C. Touati, Quasi-optimal bandwidth allocation for multi-spot MFTDMA satellites, in Proc. IEEE INFOCOM 2005, Miami, FL, 2005.

[2] F. Havet. Channel assignement and multicolouring of the induced subgraphs of the triangular lattice. Discrete Mathematics 233:219-231, 2001.

[3] F. Havet, R. J. Kang and J.-S. Sereni. Improper colourings of unit disk graphs. Electronic Notes in Discrete Mathematics 22:123-128, 2005.

[4] F. Havet, R. J. Kang and J.-S. Sereni, Improper colourings of unit disk graphs, Networks, to appear. 
[5] F. Havet and J. Zerovnik. Finding a five bicolouring of a triangle-free subgraph of the triangular lattice. Discrete Mathematics 244:103-108, 2002.

[6] J. van den Heuvel, R. A. Leese and M. A. Sheperd. Graph Labelling and radio channel assignment. J. Graph Theory 29(4):263-283, 1998.

[7] L. Narayanan and S. Schende. Static Frequency Assignment in Cellular Networks. In Sirocco 97, (Proceedings of the 4 th international Colloqium on structural information and communication complexity, Ascona, Switzerland), D. Krizanc and P. Wildmayer (eds.), Carleton Scientific pp. 215-227, 1997.

[8] C. McDiarmid and B. Reed. Channel assignment and weighted coloring. Networks 36:114-117, 2000.

[9] J.-S. Sereni. Coloration de graphes et applications. PhD thesis, University of Nice-Sophia Antipolis, July 2006.

[10] K. S. Sudeep and S. Vishwanathan. A technique for multicoloring triangle-free hexagonal graphs. Discrete Mathematics 300(1-3), 256-259, 2005. 